\title{
Mechanisms of Continence and Surgical Cure in Female and Male SUI: Surgical Research Initiatives
}

\author{
David Staskin, ${ }^{1}$ Andrea Tubaro, ${ }^{2}$ Peggy A. Norton, ${ }^{3}$ and James A. Ashton-Miller ${ }^{4 *}$ \\ ${ }^{1}$ Division of Urology, St.Elizabeth's Medical Center, Tufts University School of Medicine, Boston, Massachusetts \\ ${ }^{2}$ Department of Urology, Sant'Andrea Hospital, "La Sapienza" University of Rome, Rome, Italy \\ ${ }^{3}$ Department of Obstetrics and Gynecology, University of Utah, School of Medicine, Salt Lake City, Utah \\ ${ }^{4}$ Department of Mechanical Engineering, University of Michigan, Ann Arbor, Michigan
}

\begin{abstract}
Aims: To report the conclusions of the Think Tank on mechanisms of incontinence and surgical cure in female and male SUI: surgical research initiatives during the ICI-RS meeting in 2010. Methods: The sub-group considered five areas for future research in stress urinary incontinence (SUI); (i) epidemiology and public health efforts in SUI, (ii) the basic sciences examining the physiology and pathophysiology of the continence mechanism, (iii) diagnostic techniques and clinical assessment of SUI, (iv) the future of treatment and surgical cure, and (v) the separate issue of male SUI. Results: Roadblocks to progress were identified for each of the five directions. Conclusions: Future research directions are suggested for each of these areas. Neurourol. Urodynam 30:704-707, 2011. (c) 2011 Wiley-Liss, Inc.
\end{abstract}

Key words: diagnosis; epidemiology; treatment; urinary incontinence

\section{INTRODUCTION}

Stress urinary incontinence (SUI) is defined as the loss of urine with effort (an increase in intra-abdominal pressure which overcomes the urethral resistance in the absence of a true bladder contraction). The entire group who elected this Think Tank identified areas of future research, purposely inclusive of a broad range of research interests; epidemiology and public health, pathophysiology, diagnosis, treatment, and the male patient. The inclusion of the male patient was based on the perception by the group of a relatively disproportionate historical focus on the female patient in the SUI literature, particularly in the basic sciences. Each sub-group identified an area or areas of interest within their topic based on a balance between the perceived need for further research and the available knowledge base.

\section{EPIDEMIOLOGY AND PUBLIC HEALTH EFFORTS IN SUI}

Advances in epidemiology are required to prioritize research into the utilization and development of both current and evolving treatment options. Surgical research must be able to identify and understand the population at risk. The threshold for treatment of SUI differs depending on cultural norms, the source of payment for various treatments, and expectations of outcome. Representatives from disparate geographic medical systems agree that all countries are struggling with algorithms to best utilize available resources. Simple questions persist about which patient benefits from which treatment. There is an acknowledged disconnect between objective severity of SUI (frequency of incontinence, volume of urine loss) and the subjective bother to the individual. Co-morbidities such as obesity and diabetes are increasing and may have deleterious effects on the management of SUI. A "needs assessment" instrument is essential for creating an accurate resource allocation for the types of therapy that will be required by evolving sub-populations (e.g., aging population and those patients with concomitant co-morbidities).

Little progress has been made in the prevention of SUI. Because SUI is common in women, has a significant effect on quality of life, and is not always treated successfully, therefore prevention of SUI (primary, secondary, and tertiary) should be a higher priority. The contribution of family history has been found to play an important role in SUI, and genetics may have contributions to the development of SUI equal to that of environment; approximately $40 \%$ has been ascribed to each according to twin studies. We may be able to use enriched study populations (i.e., higher risk groups such as those with a family history) to facilitate prevention studies.

\section{THE BASIC SCIENCES EXAMINING THE PHYSIOLOGY AND PATHOPHYSIOLOGY OF THE CONTINENCE MECHANISM}

A better understanding of the pathophysiology of SUI is needed specifically by expanding basic science research of the continence mechanism. While many factors can contribute to SUI, we shall argue that recent research suggests that one of the most important is maximum urethral closure pressure (MUCP). There is generally a wide variation in MUCP in healthy continent nullipara. For example, in the third decade MUCP averages $92 \mathrm{~cm} \mathrm{H}_{2} \mathrm{O}$ and ranges from about 70 to $115 \mathrm{~cm} \mathrm{H}_{2} \mathrm{O}$, but by the seventh decade the effect of age has caused it to decrease to an average of $37 \mathrm{~cm} \mathrm{H}_{2} \mathrm{O}$ and few values exceed $50 \mathrm{~cm} \mathrm{H}_{2} \mathrm{O}^{1}$ While part of this age-related loss in MUCP can probably be attributed to an age-related loss in smooth muscle cell density observed, ${ }^{2}$ some may be caused by the age-related loss of striated urethral muscles cells ${ }^{3,4}$ as well as the urethral nerve axons. ${ }^{5}$ While these in vitro studies are valuable, there remains the need to directly correlate urethral

Eric Rovner led the review process

Conflicts of interest: Staskin- AMS: Owner of patent, consultant, royalties, speaker honorarium. Ashton-Miller- AMS: Consultant, research grant; Kimberly Clark Corp: Research grant.

*Correspondence to: James A. Ashton-Miller, PhD, Biomechanics Research Laboratory, G.G. Brown Laboratories, Department of Mechanical Engineering, University of Michigan Ann Arbor, MI 48109-2125, USA. E-mail: jaam@umich.edu

Received 20 February 2011; Accepted 17 March 2011

Published online 15 June 2011 in Wiley Online Library

(wileyonlinelibrary.com).

DOI 10.1002/nau.21139 
striated and smooth muscle volume and quality with MUCP in vitro. Present ultrasound and MR techniques will need to be further improved before this can be done reliably. In parous women there is weak histological evidence that some of the loss in urethral striated muscle and nerve is related to parity. ${ }^{3-5}$ And it is known that parity adversely affects MUCP: for example, the MUCP in primipara with de novo SUI average $63 \mathrm{~cm} \mathrm{H} \mathrm{H}_{2} \mathrm{O}$ compared with $90 \mathrm{~cm} \mathrm{H}_{2} \mathrm{O}$ in nulliparous continent controls. ${ }^{6}$ This is important because decreased MUCP is now known to be a slightly stronger contributor to SUI than urethral hypermobility. ${ }^{7}$ From a functional standpoint one knows that the lower the MUCP the higher the risk of SUI, which occurs whenever intra-abdominal pressure exceeds MUCP. ${ }^{8}$ Hence, one might expect that a nullipara starting adult life with a low MUCP is more likely to leak when she is older than a nullipara with a high initial MUCP; but this remains to be proven. Racial differences in MUCP have been found: whites average 22\% lower MUCP than blacks $(56 \mathrm{~cm}$ $\mathrm{H}_{2} \mathrm{O}$ vs. $\left.68 \mathrm{~cm} \mathrm{H}_{2} \mathrm{O}\right) .{ }^{9}$ Why this racial difference is present is not yet clear. Does the difference lie, for example, in the striated or smooth muscle tone or volume, or the blood pressure in the vascular plexus? What is known is that, when either group has SUI, their MUCP is about 20\% lower than normal. ${ }^{8}$

Despite the wealth of data on MUCP it is an inconvenient truth, and an opportunity for future research, that all past and current measurements of MUCP suffer from systematic measurement bias. Firstly, any published measurement of urethral closure pressure contains measurement artifact, due to the diameter of the current urodynamics catheters disturbing the physiology of what is being measured: for example, a 6 or $8 \mathrm{~F}$ catheter will part and unfold the coapted surfaces of the urethral lumen merely to gain access to the lumen, and inspection of axial MR and histological cross-sectional urethral anatomy ${ }^{10}$ would suggest that the catheter likely partially occludes the vascular plexus, and possibly lengthens the circumferentially arranged sphincteric muscle layers. The latest generation of ultraminiature pressure transducers, measuring fractions of a millimeter, would avoid some, but not all of these problems. Second, the radial and flexural stiffness of the current catheters is considerably stiffer than that of the urethral lumen, vascular plexus, and sphincter muscles. This means that it probably tends to straighten a curved urethra on the one hand, and also tend to prevent a urethra from bending naturally along its length during a cough or valsalva, on the other. Both effects can foster measurement artifact. Thirdly, the longitudinal stiffness of the catheter means that it may be difficult to eliminate movement of the urethral mucosa relative to the longitudinal axis of the catheter during a cough or sneeze. ${ }^{11}$ This particular artifact can be eliminated through use of a suction port.11 Fourthly, current urodynamics catheters fail to allow one to differentiate between fluid pressure and a contact stress applied to the sensor. The former is the urine pressure at that location, while the latter is due to the direct contact of the mucosal tissues on the pressure sensor. The two are not necessarily equivalent. Novel methods are being explored to examine urethral function, ${ }^{12}$ however, these need to be further miniaturized so as to avoid altering the very physiology that they are studying, for the reasons already given

Modern methods of tissue engineering allow one to grow scaffold-less ligament and muscle tubular structures that permit nerve and blood vessel growth along those tubes. ${ }^{13}$ However, much work remains to be done to grow anything that resembles the sophistication of a urethra complete with lumen, vascular plexus, and smooth and striated muscle layers with ability to be controlled neurologically. This brings up the question as to how much each of those layers contributes to MUCP. The current dogma, reviewed by Ashton-Miller and DeLancey, ${ }^{14}$ is that the vascular layer, smooth muscle, and striated muscle layers each contribute 33\% to MUCP. However, the data are based on a small study using older methods. Given the importance of low MUCP to SUI, a current knowledge gap is how much each of these layers contribute to MUCP in health, age, and disease. What exactly underlies, for example, the loss in MUCP, discussed above, that has been documented with age and parity? The answer would help direct future tissue engineering efforts by identifying tissue impairment causing the greatest deficit in MUCP.

Lastly, much is known about the neural control of the rodent urethra, ${ }^{15}$ but less is known about the control of the human urethra and pelvic floor in vivo. In vivo studies are starting to be performed ${ }^{16}$ but more are needed. What function, for example, does the longitudinal smooth muscle of the urethra have? Is it involved in the initiation of micturition by exerting tension to help open the vesical neck? Finite element models might give useful insights in this regard.

\section{DIAGNOSTIC TECHNIOUES AND CLINICAL ASSESSMENT FOR SUI}

An accurate diagnosis is essential for the implementation of effective and efficient treatment algorithms in SUI, as well as the subjective and objective assessment of treatment outcomes. However, the sub-group recognizes the need to prioritize these research goals due to the limited resources in less developed countries as well as the potential for limits on future health research expenditures in any country. Complex testing such as urodynamics is widely recommended for the assessment of SUI, even though the equipment required is expensive and not readily available. The concept that "the bladder is an unreliable witness" stemmed from the lack of correlation between patient symptoms and the results of such high tech testing. The testing was said to be of more consequence than the patient's own complaints, but there is little evidence that such testing is needed prior to surgery for SUI (see sub-committee statement on "urodynamics"). The subcommittee recommended that no further clinical tests should be utilized in the routine evaluation of SUI without evidence of its usefulness, especially those involving significant expense or discomfort to the patient. Current clinical testing should continue to be subjected to these standards of evidence-based utility, and a high priority is to develop clinical testing that predicts which patients benefit from which treatments.

\section{THE FUTURE OF TREATMENT AND SURGICAL CURE}

Priorities in surgical treatment were detailed in the 1st ICIRS and include (i) the creation of large-scale collaborative databases designed to foster high-quality data collection, (ii) the development of a system of standardized outcome measures, (iii) the selection of treatments/procedures to be placed in the database, and (iv) the establishment of an organizational structure to be responsible for instituting the standards and procedural mechanisms to ensure the integrity of the data analysis. ${ }^{17}$ It was agreed by the committee that the first surgery is likely to yield the best results, and that all secondary treatments are likely to be less effective. Thus, the concept of surgical cure must take into account whether the treatment is primary or secondary. Reimbursement for treatment of SUI in most countries is NOT based on treatment outcomes. Much could be learned by concentrating on surgical failures. This sub-group concentrated on the need for 
research that will improve the metrics for evaluating patient expectations prior to, and satisfaction after, surgical intervention.

There is a lack of data regarding patient expectations for treatment of SUI. In addition, there is considerable evidence that there may be a discrepancy between patient satisfaction and objective outcome measures. Compared to currently favored quality of life measures, an individual-patient goaldriven approach which uses composite endpoints may more clinically meaningful. ${ }^{18-22}$

While disease specific OoL questionnaires allow broad comparisons to be made assessing patient bother they may lack the sensitivity to assess individual symptoms. Isolated "objective" measures such as standard volume stress test and urodynamics are less likely to document stress leakage in women, and when used as the primary endpoint in a surgical trial, may yield more favorable "success rates" compared to subjective outcomes. ${ }^{23}$ Composite outcomes encompassing multiple objective and subjective measures to define treatment success are likely to have lower "success rates" in the same individual. Comparison between studies would be facilitated by the use of a single composite measure. Increased use of databases to compare outcomes and adverse events (AE) is advocated.

The group identified as a high priority the ability to improve communication between the physician and patient, including an improved understanding of physician-based outcomes (e.g., safety, morbidity, efficacy, efficiency) and patient-centered outcomes (e.g., patient preferences, expectations, and satisfaction). In addition, the committee identified the need for research into alternative therapies for the treatment of SUI, including new surgical techniques and new implant materials for stress incontinence surgery which are developed in parallel with defined pathways for introducing these therapies into general clinical practice. Communicating and translating the surgeon's expectations of treatment outcomes into the patient's set of expectations will improve the informed consent process.

There is general agreement that many efficacy outcomes remain poorly defined. ${ }^{17}$ However, equally as critical, the definition and classification of $\mathrm{AE}$ and the "morbidity-impact" of these specific AEs also requires further research. Research is needed to understand the impact of specific AEs on the individual patient as opposed to the incidence of the $\mathrm{AE}$ in a population-and the relative impact of the specific AE to the presurgical condition of the individual patient. The terminology to classify AEs is deficient as descriptors such as "pre-op" also do not reflect the impact or bother of the AE, especially with respect to the trade-off between the "de novo" or worsened post-operative symptoms and the desired and perceived improvement from the surgery. Due to the need to understand the incidence and impact of AEs, and given the possibility of limited resources, the committee suggested that a complications registry with a known denominator would be of the most immediate benefit to patient care, although a comprehensive data registry that also details efficacy is unquestionably preferable.

Specific to urinary incontinence is the interrelationship between stress and urgency incontinence (or OAB dry symptoms). In general, a patient who has residual SUI but is relieved of urgency symptoms will be more satisfied with treatment than a patient who is completely cured of SUI and has residual urgency symptoms, and certainly more than the patient who is cured of SUI and develops urgency incontinence even though the surgery was for SUI (consensus agreement by the sub-committee). Clinically, this means that women with mixed stress and urgency incontinence are more difficult to treat because both components of their leakage must improve for the patient to achieve continence.

\section{THE SEPARATE ISSUE OF MALE SUI}

Male urinary incontinence has been a rather neglected research topic in comparison to the condition in females. This is in part because of its iatrogenic nature possibly because of the natural tendency of surgeons treating prostate cancer to minimize the morbidity of male USI in lieu of the primary condition they are treating. This surgical sub-group considered areas for future research in the classification, diagnosis, and treatment of stress incontinence in the male patient.

Male patients may suffer different forms of urinary incontinence including urgency incontinence, primary stress incontinence (rare), iatrogenic stress incontinence following prostate surgery, UI associated with chronic retention (once called overflow incontinence), and urinary incontinence associated with post-micturition dribbling. The prevalence of SUI in the male population is known to be lower than in female cohorts $^{24}$ primarily because the high outlet resistance of the male urinary tract makes leakage a rare event. Epidemiology studies suggest that men are severely bothered by $\mathrm{SUI}^{24}$ and they find it difficult to cope with the condition. This is also because SUI is generally considered by clinicians to be a female issue, and pads are not in the men's armamentarium for other conditions. Interestingly, incontinent men seem to suffer a larger emotional impact than women. ${ }^{25}$

Primary stress incontinence in men is rare and is usually observed in neurogenic patients with impaired sphincter function or in frail elderly patients. ${ }^{26}$ The prevalence of the condition is rare enough to make clinical research a low priority.

Iatrogenic urinary incontinence, of a degree that necessitates invasive therapy, is a less common but serious complication of simple prostatectomy and transurethral resection of the prostate. ${ }^{27}$ The condition is considered to be due to sphincter damage. Endoscopic imaging of incontinent patients usually reveals scar tissue in the dorsal part of the external sphincter and although active recruitment often results in proper closure mechanism, the sphincter remains open at rest. No major clinical questions remain open on this subject beyond the need to provide continence rate for any new surgical procedures that becomes available for the management of BPO.

Urinary incontinence following radical prostatectomy is the most prevalent form of iatrogenic incontinence in urology. ${ }^{28}$ Incidence rate depend upon the definition of continence (e.g., dry or no pads vs. a single protection pad), and in case series varies between $10 \%$ and $50 \%$ at 1 year from surgery. ${ }^{29}$ No consensus exists on the mechanisms underlying SUI following radical prostatectomy and whether there are any preoperative risk factors. ${ }^{29}$ Both sphincter and detrusor dysfunction can play a role. SUI is clearly dependent upon sphincter incompetence but it is still unclear what is the relative contribution of neural damage, direct damage of muscle fibers, and sphincter dislocation. A number of studies investigated preoperative sphincter parameters such as MUCP and sphincter length trying to identify threshold values associated with a higher risk of SUI but the case remains open. Careful dissection of the prostate apex and preservation of the neural bundles seem to be associated with increased continence rates suggesting that sphincter denervation may be of importance although the data remain conflicting. ${ }^{30,31}$ Surgery of post radical prostatectomy incontinence with the Advance sling results suggests that downward dislocation of the sphincter complex may be 
responsible for suboptimal closure mechanism although definitive proof of the concept is still lacking. ${ }^{32}$

Since 2002, when the change in terminology was adopted, use of the old term "overflow incontinence has been discontinued." Although the terminology might have been inaccurate, the condition remains real and patients sometimes present with chronic retention and SUI due to volume overload of the urinary bladder. Research is certainly needed in this area to identify a correct terminology to define this condition.

\section{CONCLUSIONS}

A number of academic questions remain open and certainly deserve properly designed, carefully conducted studies with adequate funding. The research priorities for SUI are listed below,

(i) Identifying the epidemiology and promoting public health efforts: specifically, improving knowledge of the characteristics of the "at risk" population, understanding resources, and investigating methods of prevention.

(ii) Advancing the basic science understanding of the continence mechanism.

(iii) Improving diagnostic and clinical techniques.

(iv) Interpreting outcomes of surgical treatment: specifically, re-examination of the metrics for determining patient bother and the metrics for surgical outcomes-including patient-driven expectations and goals.

(v) Expanding recognition and treatment of the male patient: specifically, acknowledging the burden of disease of SUI in the male population and applying the research initiatives above (i-iv) to the male as well as the female population.

\section{REFERENCES}

1. Trowbridge RE, Wei JT, Fenner DE, et al. Effects of aging on lower urinary tract and pelvic floor function in nulliparous women. Obstet Gynecol 2007;109:715-720

2. Clobes A, DeLancey JO, Morgan DM. Urethral circular smooth muscle in young and old women. Am J Obstet Gynecol 2008;198:e1-e5.

3. Perucchini D, DeLancey JOL, Ashton-Miller JA, et al. Age effects on urethral striated muscle: I Changes in number and diameter of striated muscle fibers in the ventral urethra. Am J Obstet Gynecol 2002;186:351-355.

4. Perucchini D, DeLancey JOL, Ashton-Miller JA, et al. Age effects urethral striated muscle: II. Anatomic location of muscle loss. Am J Obstet Gynecol 2002;186:356-360.

5. Pandit M, DeLancey JO, Ashton-Miller JA, et al. Ouantification of intramuscular nerves within the female striated urogenital sphincter muscle. Obstet Gynecol 2000;95:797-800.

6. DeLancey JO, Miller JM, Kearney R, et al. Vaginal birth and de novo stress incontinence: Relative contributions of urethral dysfunction and mobility. Obstet Gynecol 2007;110:354-362.

7. DeLancey JOL, Trowbridge ER, Miller JM, et al. Stress urinary incontinence: Relative importance of urethral support and urethral closure pressure. J Urol 2008;179:2286-2290

8. Kim K-J, Ashton-Miller JA, Strohbehn K, et al. The vesico-urethral pressure gram analysis of urethral function under stress. J Biomech 1997;30:19-25.
9. DeLancey JOL, Fenner DE, Guire K, et al. Differences in continence system between community-dwelling black and white women with and without urinary incontinence in the EPI study. Am J Obstet Gynecol 2010;202: e1-e12.

10. Strohbehn K, Quint LE, Prince MR, et al. Magnetic resonance imaging anatomy of the female urethra: A direct histologic comparison. Obstet Gynecol 1996;88:750-756.

11. Stafford RE, Sapsford R, Ashton-Miller JA, et al. A novel transurethral surface electrode to record male striated urethral sphincter electromyographic activity. J Urol 2010;183:378-385.

12. Klarskov N, Lose G. Urethral pressure Re£ectometry; A novel technique for simultaneous recording of pressure and cross-sectional area in the female urethra. Neurourol Urodyn 2007;26:254-261.

13. Ma J, Goble K, Smietana $M$, et al. Morphological and functional characteristics of three-dimensional engineered bone-ligament-bone constructs following implantation. J Biomech Eng 2009;131: art. no. 101017-1.

14. Ashton-Miller JA, DeLancey JOL. Functional anatomy of the female pelvic floor. Ann NY Acad Sci 2007;1101:266-296.

15. Thor KB, deGroat WC. Neural control of the female urethral and anal rhabdosphincters and pelvic floor muscles. Am J Physiol Regul Integr Comp Physiol 2010;299:R416-R438.

16. Junginger B, Baessler K, Sapsford R, et al. Effect of abdominal and pelvic floor tasks on muscle activity, bladder pressure and bladder neck. Int Urogyn J 2010;21:69-77.

17. Staskin D, Khullar V. Female pelvic surgical devices and techniques need better evidence-based medicine. Neurourol Urodyn 2010;29:670-675.

18. Tincello DG, Alfirevic Z. Important clinical outcomes in urogynaecology: Views of patients, nurses and medical staff. Int Urogynaecol J 2002;13:96.

19. Elkadry EA, Kenton KS, Fitzgerald MP, et al. Patient selected goals: A new perspective on surgical outcome. Am J Obstet Gynaecol 2003;189:15511557.

20. Mahajan ST, Elkadry EA, Kenton KS, et al. Patient centered surgical outcomes: The impact of goal achievement and urge incontinence on patient satisfaction one year after surgery. Am J Obstet Gynaecol 2006;194:722728.

21. Lowenstein L, Fitzgerald MP, Kenton K, et al. Patient-selected goals: The fourth dimension in assessment of pelvic floor disorders. Int Urogyn J Pelvic Floor Dysfunct 2008;19:81-84.

22. Srikrishna S, Robinson D, Cardozo L. Qualifying a quantitative approach to women's expectations of continence surgery. Int Urogyn J Pelvic Floor Dysfunct 2009;20:859-865.

23. Ward JF, Sebo TJ, Blute ML, et al. Salvage surgery for radiorecurrent prostate cancer: Contemporary outcomes. J Urol 2005;173:1156-1160.

24. Kwong PW, Cumming RG, Chan L, et al. Urinary incontinence and quality of life among older community-dwelling Australian men: The CHAMP study. Age Ageing 2010;39:349-354

25. Teunissen D, Van Den Bosch W, Van Weel C, et al. "It can always happen": The impact of urinary incontinence on elderly men and women. Scand J Prim Health Care 2006;24:166-173.

26. Miu DK, Lau S, Szeto SS. Etiology and predictors of urinary incontinence and its effect on quality of life. Geriatr Gerontol Int 2010;10:177-182.

27. Rassweiler J, Teber D, Kuntz R, et al. Complications of transurethral resection of the prostate (TURP)-incidence, management, and prevention. Eur Urol 2006;50:969-979.

28. Jacobsen NE, Moore KN, Estey E, et al. Open versus laparoscopic radical prostatectomy: A prospective comparison of postoperative urinary incontinence rates. J Urol 2007; 177:615-619.

29. Sacco E, Prayer-Galetti T, Pinto F, et al. Urinary incontinence after radical prostatectomy: Incidence by definition, risk factors and temporal trend in a large series with a long-term follow-up. BJU Int 2006;97:1234-1241.

30. Potdevin L, Ercolani M, Jeong J, et al. Functional and oncologic outcomes comparing interfascial and intrafascial nerve sparing in robot-assisted laparoscopic radical prostatectomies. J Endourol 2009;23:1479-1484.

31. Toren P, Alibhai SM, Matthew A, et al. The effect of nerve-sparing surgery on patient-reported continence post-radical prostatectomy. Can Urol Assoc J 2009;3:465-470.

32. Bauer RM, Mayer ME, Gratzke C, et al. Prospective evaluation of the functional sling suspension for male postprostatectomy stress urinary incontinence: Results after 1 Year. Eur Urol 2009;56:928-933. 